Volume 10, No.3, May - June 2021

International Journal of Advanced Trends in Computer Science and Engineering

Available Online at http://www.warse.org/IJATCSE/static/pdf/file/ijatcse251032021.pdf

https://doi.org/10.30534/ijatcse/2021/251032021

\title{
Smart Grid Local Energy Trading Based Crypto Token Using Blockchain
}

\author{
THARUN $\mathbf{P}^{1}$, SHAHRUKH PARVEZ $\mathbf{R}^{2}$ \\ ${ }^{1}$ St Joseph's College of Engineering, India, tharunfutur@ outlook.com \\ ${ }^{2}$ St Joseph's College of Engineering, India, shahrukhparvez10431@gmail.com
}

\begin{abstract}
Smart grid is envisioned to be the technology capable of scheduling user's energy requirement based on demand and decentralized nature. These challenges pose extreme pressure on finding advanced technologies and sustainable solutions for secure and reliable operations of the power system working inside the blockchain technology for managing exchange and trading of energy by means of specific tokens. For efficient utilization and functioning of the power grid we need a decentralised system which is transparent, trustless and makes transactions faster, there are a number of solutions proposed but none of them address the issue of transaction time in trade and penalty for defaulters. In this work we propose here an energy transaction network which implements blockchain technology for validating transaction of energy between producer/consumer or prosumer and saves energy and time using smart grids.
\end{abstract}

Key words : Energy trading, Blockchain, Smart contracts, Smart grid, IPFS, Energy trading, Distributed Energy Resources, Microgrid, Security.

\section{INTRODUCTION}

Smart grids are a digitally automated technology capable of controlling, monitoring and analyzing the flow and requirement of energy according to the market need. Unlike the traditional electricity grid, smart grids us es two-way flow of electricity and information for an automated and advanced energy delivery network.[1] These are expected to increase efficiency of utilization of energy, cost effective and environmental friendly [2].

The challenge is to create a trading system that captures the interest and answers the needs of prosumers and also provides a data infrastructure that meet the necessity of electric service operators to govern the energy grid. For a stable and cost effective grid operation demand and response should be balanced since production of peak energy and storage of excess energy is expensive [3].

Hence it is the responsibility of all grid participants to dynamically manage energy consumption. Smart grids connected through smart meter keep track of energy flow between suppliers and producer/consumer or prosumers using enables the supply and demand sides to interact with each other by exchanging the price and demand information to make better decisions [5] based on the blockchain technology, token price refers to the current price of a cryptocurrency token in their own fiat money.

As said, to insert images in Word, position the cursor at the insertion point and either use Insert | Picture | From File or copy the image to the Windows clipboard and then Edit | Paste Special | Picture (with "Float over text" unchecked).

- The main objective of this paper is to illustrate how blockchain smart contracts can be used apace with IPFS to introduce a decentralized, credible, trusted smart grid solar energy system. The main contributions of this paper can be summarized as follows: We proposed a blockchain based solution using smart contract and IPFS for a smart grid solar energy system that is highly reliable, secure, decentralized, and trusted. The system allows tokens to be issued to valid consumption of energy and have them paid upon successful of consumption of energy.

- Efficiency starts with information its now between the consumer and producers. Hence a two-way communication system that shows the actual energy usage is important. Based on this consumer may reduce their energy cost by shifting their load from periods of high prices to periods of lower prices.

- We present details on system overview, design, architecture, and the interactions of the various actors in the system.

- We provide a complete implementation of the smart contract code and discuss key aspects related to the backend interfaces used by actors.

The remainder of the paper is organized as follows. Section II gives some background information on smart contracts and blockchain. Section III summarizes the related work that exists in the literature. Section IV presents our proposed blockchain based smart grid system solution. 
THARUN P et al., International Journal of Advanced Trends in Computer Science and Engineering, 10(3), May - June 2021, 1663 - 1670

\section{BLOCKCHAIN METHODOLOGY}

\section{A. Blockchain}

A blockchain database is a collection of information that is stored electronically on a computer system. Information, or data, in databases is typically structured in table format to allow for easier searching and filtering for specific information. What is the difference between someone using a spreadsheet to store information rather than a database One key difference between a typical database and a blockchain is key difference between a typical database and a blockchain is the way the data is structured. A blockchain collects information together in groups, also known as blocks, that hold sets of information. Blocks have certain storage capacities and, when filled, are chained onto the previously filled block, forming a chain of data known as the "blockchain." All new information that follows that freshly added block is compiled into a newly formed block that will then also be added to the chain once filled.

\section{B. Smart Contract}

A "Smart Contract" is simply a program that runs on the Ethereum blockchain. It's a collection of code (its functions) and data (its state) that resides at a specific address on the blockchain. Smart contracts are a type of Ethereum account. This means they have a balance and they can send transactions over the network. However, they're not controlled by a user, instead they are deployed to the network and run as programmed. User accounts can then interact with a smart contract by submitting transactions that execute a function defined on the smart contract. Smart contracts can define rules, like a regular contract, and automatically enforce them via the code.

In other words, smart contract is a computer code that runs on top of a distributed ledger and includes rules that the involved parties have agreed upon in order to interact with each other. In the case that these rules are satisfied, the smart contract agreement is automatically executed and cannot make any changes or modifications to it. The main feature of this smart contract is that when a condition is met, or an event is reached, the contract can be executed automatically according to the scripts.

\section{IPFS}

IPFS (the Inter Planetary File System) is a hypermedia distribution protocol addressed by content and identities. It enables the creation of completely distributed applications, and in doing so aims to make the web faster, safer, and more open. IPFS is a distributed file system that seeks to connect all computing devices with the same system of files. In some ways, this is similar to the original aims of the Web, but IPFS is actually more similar to a single BitTorrent swarm exchanging git objects. In addition, due to its decentralized nature, there isn't a single point of failure i.e., if one device got disconnected. The file still can be accessed.

\section{TOKEN TRADING}

Energy token is spendable and tradeable just like a cryptocurrency but focused on incentivizing real-world environmental activity: verifiably produced solar energy. Energy token is a reward for solar energy producers. The energy token gives energy producers blockchain-based digital tokens at the rate of one energy token (ET) per Megawatt-Hour (MWh) of solar energy produced. Energy token is free. It is an additional reward for solar energy producers.

\section{RELATED WORK}

To understand the significance of the given problem that we need to understand demand response (DR) and how demand side management is integrated into smart grids. Present power grid system provides exceed rate electricity to most consumers with no incentives to adjust energy consumption which could improve energy. Efficiency and power system reliability. DR is a system in which customers can react to price variation which re acts real operational costs and hence can bring relevant savings in system operation. For example, some portion of the demand in times of high generation costs may be avoided or shifted to less-expensive periods. It facilitates real-time balance of supply and demand and is considered as a main option to overcome the problems caused by variable and uncertain output of renewable energy sources [9], [10]. Hence following are some of the characteristics of a system capable of implementing it as discussed in [9][11][12]. Efficiency starts with information flow between the consumer and producers. Hence a two-way communication system that shows the actual energy usage is important. Based on this consumer may reduce their energy cost by shifting their load from periods of high prices to periods of lower prices. With usage information comes the need for optimal scheduling mechanism to regulate energy usage based on price and emergency. Success of it depends on the active participation of consumers. To encourage con-summers an incentive-based system benefiting all parties is required.

Innovative smart technologies like smart meters, smart thermostats integrated with smart management system enables better management of energy. Basic concept of smart grid and technologies used are explained in [2][3][4][13][14]. In [15] the authors discuss communication architectures for advanced metering infrastructure (AMI) in smart grid and [16] gives the framework of an architecture for appropriate smart control system. Authors of [17][18] provides details on various communication mechanisms used between smart me-term and consumer equipment through various wireless 
networking solutions like ZigBee and, these papers address the rest of other characteristics of the system capable of implementing [19] propose a practical pricing scheme to encourage die rent consumers to participate in demand response by providing them with a list of price plans. Upon classifying each consumer based on individual information a pricing model is formulated as a nonlinear programming problem, aiming to minimize the overall operation cost. Authors of [20] targets at minimizing peak-to-average ratio (PAR) of power usage in the grid with a centralized scheme. Further proposes a scheme targeting minimum power generation cost to motivate power suppliers to join the DR system and a game theoretical approach that benets all parties at same level. Authors of [21][22] also proposes differ-net game theoretical approaches for residential customers based on dynamic smart pricing.[23] proposes a DSM method consisting in a day-ahead optimization process.

The main objective stated in this work is to reduce user's monetary expense during the time period of analysis by producing and/or storing energy rather than just purchasing user energy needed from the grid. Authors of [24] investigates optimal energy management for the SG, taking into consideration unpredictable load demands and distributed energy re-sources. In [25] the authors propose a distributed algorithm to attend power-demand curve at transformers to avoid overloading and defer investment. Although these techniques im-prove PAR of energy consumption and reduce cost for producers, the implementation of these optimal pricing technique requires a decentralized system.

\section{BLOCKCHAIN IN DISTRIBUTED TRANSACTION}

The advancement in various communication mechanisms and large data exchanges makes central management and operation more and more challenging. Local distributed control and management techniques are required for smooth operation of the smart grid. Centralized systems also have disadvantages due to a single point of failure, which makes them more vulnerable to both technical failures and malicious attacks. Blockchains or distributed ledger technologies (DLT), were primarily designed to facilitate distributed transactions by removing central management[7]. Blockchains are shared and distributed data blocks cryptographically chained in chronological order [26]. Authors of [27] [28] gives a review on the working mechanism of blockchains and smart contracts. Blockchains are difficult to tamper with unless a significant part of the network is colluding. Usage of hash functions and public-key cryptography ensures enhanced security in blockchain systems. In the paper [29] the authors investigate the use of decentralized blockchain mechanisms for delivering transparent, secure, reliable, and timely energy flexibility, under the form of adaptation of energy demand proles of
Distributed Energy Prosumers, to all the stakeholders involved in the exibility markets. Authors of [30][7][8] gives details on the significance, challenges and opportunities of blockchain technology in the energy sector. Major challenges pointed out on these surveys are scalability and speed. Proof of Work (PoW) algorithms used in mining blocks are slow and very energy intensive. Alternate algorithms like Proof of Stake (PoS), Practical Byzantine Fault Tolerance (PBFT), Delegated Proof of Stake (DPoS), Proof of Activity (PoAc) etc are adopted at the expense of security and decentralization. Authors of [31] discusses a sovereign blockchain-based system implemented on a smart grid creating a tamper-proof system for protecting consumer data recorded and transferred. The utilization of smart contracts in the model enhances the transparency between participants. In paper [32] the authors addressed the problem of providing transaction security in levels of privacy and security. All these works agree with the fact that blockchain based smart grid network improves efficiency and performance of a smart grid.

\section{SYSTEM ARCHITECTURE, DESIGN AND IMPLEMENTATION}

\section{A. System Overview and Design}

This is a framework consisting of a public blockchain network and power utility network. There are two entities involved in an energy network. One the actual or physical energy transfer and other is the monetary transactions. The physical energy transfer is maintained by the power utility network with smart grid which utilizes smart meters to validate energy added to and consumed from the grid. The public blockchain network is a decentralized network with power utility being one of the participating mining node. Each block is mined and added to the chain in the public utility network. The power utility network need only to keep track of opening and closing of a transaction between the producer and consumer in order to physically transfer the energy. Energy coin transaction agreement is drawn between the producer and consumer through lightning network channel and financial settlement done on the public blockchain network when the block is mined. The overview of the network is shown in Fig. 3.1. The make sure that the physical power utility network connected to the smart grids and all prosumers connected to this network through smart meters. As physical transfer requires the already established physical lines power utility network has a node in the public blockchain network. This shows all participants, wallet created by producer, consumer and utility node, and channel established between producer and consumer.

The proposed solution focuses only on using smart contracts to create a blockchain based smart grid decentralized overview of architecture this also describes different actors take part in the system. Which can be traded in any energy marketplaces. Before accessing the power grid, each prosumer needs to register their respective smart meters with 
the utility network. During registration of devices the utility network generates the user public and private key along with initial incentive energy coins.

The physical power utility network connected to the smart grids and all prosumers connected to this network through smart meters. As physical transfer requires the already established physical lines power utility network has a node in the public blockchain network. The all participants, wallet created by producer, consumer and utility node, and channel established between producer and consumer. Before accessing the power grid, each prosumer needs to register their respective smart meters with the utility network. During registration of devices the utility network generates the user public and private key along with initial incentive energy coins. Those energy coins can be stored anywhere (i.e.) crypto wallets or in the trading marketplaces.

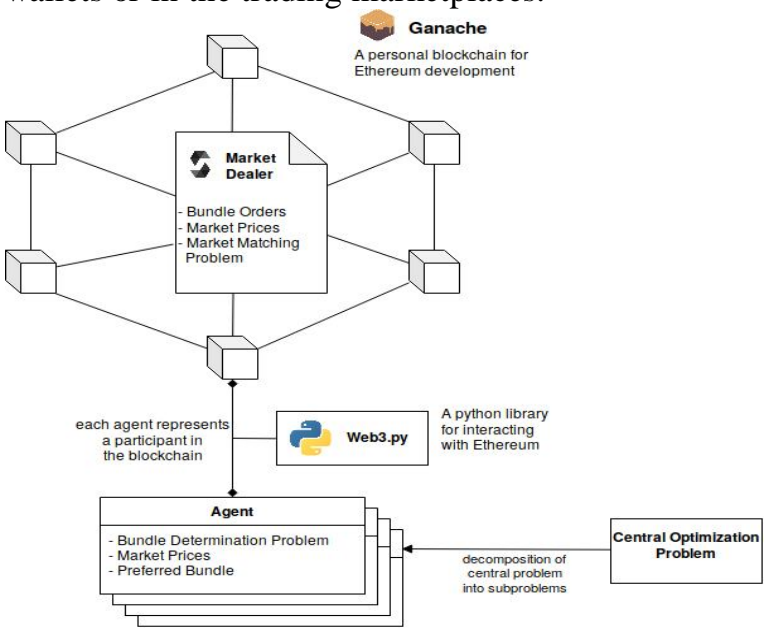

Figure 5.1: Overview of the proposed network.

\section{B. Implementation Details}

Once initiated channel establishment is the phase where lightning network protocols are used to establish a channel for transaction between producer and consumer [39]. A funding transaction which is a 2-of-3 multisig wallet, is made between the producer, consumer and utility company which is broadcasted to the public blockchain network. This is a wallet created with a time constraint $\backslash \mathrm{t} "$ decided by the utility network on the main blockchain with the consensus of the producer and consumer to be spent at a later time. By making a 2-of-3 multi-sig wallet the utility company does not become custodian escrow but determines who should get what amount in the case of non-cooperation. For the proposed network the producer can make false claim of adding energy to the grid, in such a case of dispute where the utility company is at loss, coins in the producer's wallet goes into the utility company's wallet. This is a penalty function imposed to maintain fairness in trading. All physical energy transfer can be verified through the smart meter connected to the power utility network. The funding transaction indicates the amount of energy required from the smart grid and becomes an indicator to the amount that needs to be generated to meet the market demand. The time constrain is imposed to control the price fluctuation in the market. Based on this demand the price of each energy units is published in terms of energy coins every $\mid t "$ time by the utility network. Now as there are coins reserved the producer and consumer can make any number of transactions by making commitment transactions. Whenever either of the parties decide to close the channel the nil commitment transaction can be broadcasted to the public blockchain network and the balances are settled from the multi signature wallet.

\section{Abbreviations and Acronyms}

\begin{tabular}{l|l}
\hline \hline Acronym & Their Definition \\
\hline SG & Smart Grid \\
\hline EI & Energy Internet \\
\hline IoE & Internet of Energy \\
\hline DER & Distributed Energy Resources \\
\hline EV & Electric Vehicle \\
\hline V2G & Vehicle to Grid \\
\hline SCADA & Supervisory Control and Data Acquisition \\
\hline CPS & Cyber-Physical System \\
\hline DSO & Distributed System Operator \\
\hline ESCO & Energy Service Company \\
\hline PMU & Phasor Measurement Unit \\
\hline AMI & Advanced Metering Infrastructure \\
\hline DG & Distributed Generator \\
\hline DLT & Distributed Ledger Technology \\
\hline DAG & Directed Acyclic Graph \\
\hline PoW & Proof of Work \\
\hline PoS & Proof of Stake \\
\hline BFT & Byzantine Fault Tolerance \\
\hline P2P & Peer to Peer \\
\hline ECC & Elliptic Curve Cryptography \\
\hline ICS & Industrial Control System \\
\hline ESU & Energy Storage Unit \\
\hline ADMM & Alternating Direction Method of Multipliers \\
\hline TES & Transactive Energy System \\
\hline CDA & Continuous Double Auction \\
\hline DApp & Decentralized Application \\
\hline &
\end{tabular}

\section{Wallet initialization}

The value of energy is calculated by demand-based pricing by the utility company. The factors that contribute are the present energy demand, and cost of generation and maintenance. Think Assuming once the physical power utility network is set maintenance cost will be a fixed quantity, the energy value is mostly dependent on demand. Based on demand and how much excess energy needs to be produced the cost is published by the utility company every $\backslash \mathrm{t}$ " time which can be written as in

$$
\mathrm{pt}=\mathrm{a} 1 . \mathrm{D} 2 \mathrm{t}+\mathrm{b} 1 . \mathrm{Dt}+\mathrm{c} 1
$$


The price is energy coins required per unit of energy. Hence as price increases the number of coins required to buy energy also increases. If $\mathrm{Ct}$ is the number of coins, then, Where are coin price co-efficient. The increase in transaction indicates an increase in de-mand for energy which implies the number of coins required also increases. Mathematically, As cost is high consumers regulates their energy consumption reducing wastage which in turn brings down the cost. This way the energy grid usage spikes will reduce making the grid stable.

All transaction on the network are based on energy coins. A miner will have total energy coins $\mathrm{Ci} ; \mathrm{t}$ which will include coins obtained as initial incentive, mining incentive and coin obtained on selling produced energy. From this total $\mathrm{Ci}$; the producer or consumer should add an amount of $\mathrm{Cw}$ into the multisig wallet which has a time constraint $\mathrm{T}$.

$$
\mathrm{W}=\mathrm{CwA}+\mathrm{CwB}+\mathrm{CwU}
$$

where $\mathrm{CwA}=\mathrm{A}$ 's coins, $\mathrm{CwB}=\mathrm{B}$ 's coins and $\mathrm{CwU}=$ Utility company coins. $\mathrm{W}$ is the resered coins in the 2-of-3 multisig wallet on the public blockchain that can be accessed only with two signatures from any three parties. Which makes it publicly visible and inaccessible for any single party. The transaction is made with a penalty clause, in the case of forgery from the producer side producer coins are transferred to the power utility wallet.

$$
\begin{aligned}
& \mathrm{CA}=\mathrm{CwA}-\mathrm{Cc} \\
& \mathrm{CB}=\mathrm{CwB}+\mathrm{Cc} \\
& \mathrm{CU}=\mathrm{CwU}
\end{aligned}
$$

The transaction flow in wallet creation and after settlement.

Which makes it publicly visible and inaccessible for any single party. The transaction is made with a penalty clause, in the case of forgery from the producer side producer coins are transferred to the power utility wallet. Details of the penalty function.

\section{E. Transfer of Energy and Penalty}

Miners on the network checks for signatures and funds inside the channel is settled. Each miner participating in the mining process obtains incentive coins Cin based on the mining process. $\mathrm{B}$ adds the committed energy to the grid and the power utility network based on the commitment transaction will transfer energy to A. Hence in this process a producer does not require a complete infrastructure to sell energy and need only to be part of the blockchain network.

In the case where A commits Cc coins to B and B fails to deliver the energy, $\mathrm{A}$ is at a loss. Upon this condition the utility company delivers the committed energy to A and signs the transaction and the commitment transaction is broadcasted to the main blockchain. In the main blockchain the wallet coins are distributed such that B looses all the coins committed to the utility transaction. Hence the penalty function in terms of energy coins is given to each produced energy.

The extra penalty function coins added to the utility wallet are redistributed among miners as mining incentives for next time slot. In the case of $\mathrm{CP}=0, \mathrm{x} \%$ of $\mathrm{C}$ at present time slot is given as incentives.

We assume that the producers add consumer required amount of energy to the smart grid, which is routed by the utility network according to funding transaction smart meter details. This allows in real time energy transfer to the required destination. Assuming i residential prosumers, each having $n$ appliances the problem is simulated based on the mathematical modeling in chapter IV. Initial parameters like energy per appliance, starting slot and ending slot where generated randomly.

To better visualize the correspondence between demand and cost all constants are taken as 1 i.e., as in chapter III, Eq. $3.1 \mathrm{a} ; \mathrm{b} ; \mathrm{c}=1$. Also for this simulation currency to coin conversion factor, $=1$. Hence number of coins saved is equal to currency saved. Sample setting of energy and time slots for 5 appliances is as shown in Table 5.1. Two data sets with number of procumers $\mathrm{i}=100$ and $\mathrm{i}=60$ each with 5 appliances were simulated.

\begin{tabular}{|cccc|}
\hline App n & En(kWh) & Start slot & End slot \\
\hline 1 & 624 & 6 & 12 \\
\hline 2 & 357 & 7 & 12 \\
\hline 3 & 591 & 7 & 18 \\
\hline 4 & 209 & 12 & 20 \\
\hline 5 & 970 & 8 & 12 \\
\hline
\end{tabular}

Table 5.2: Randomly generated appliance settings for simulation.

\section{SIMULATION RESULTS}

In this section we discuss about the algorithm to solve the problem in Eq. 4.6. Here we assume that each customer's initial demand for the day, Eq. 4.4, and coin cost computation, Eq.3.1, is known to all prosumers. Based on this each prosumer can compute the optimal cost of energy for each time slot and the algorithm is as follows:

\section{Algorithm 1: Minimizing cost}

/Generate start time, end time,energy for appliance $n$ of customer i.

Calculate energy eni for each $t$ time slot.

begin convex optimization $\mathrm{f}$

optimization variable Rit(i,n,t)

Calculate demand

Calculate cost for each time slot

minimize(total cost) 
subject to

If $\mathrm{f}$ within given end time of an applianceg

Rit(i,n,t) eni;

Iffgreater than end timeg

$\operatorname{Rit}(\mathrm{i}, \mathrm{n}, \mathrm{t})==0$;

$\operatorname{sum}($ demand per $\mathrm{n}$ over 24 time slot $)==$ total energy required for the appliance.

$0<$ sum(demand over 24 time slot)<constant value.

$\mathrm{g}$ end optimization

Fig. 6.1 and Fig. 6.2 shows total demand on the smart grid for a day for different number of prosumers $(i=100$ and $i=60)$ and each prosumer having 5 appliances. In both data sets we can see that implementation of this system stabilizes the load on the smart grid. The peak-to-average values for both set of data along with randomly chosen individual user's values are shown in Table 5.1. Also, there is no sudden variation in demand which supports grid efficiency. This is better illustrated by the second data set which has more fluctuations in demand for sure with a PAR of 1:532 before optimization and 1:1612 after optimization.

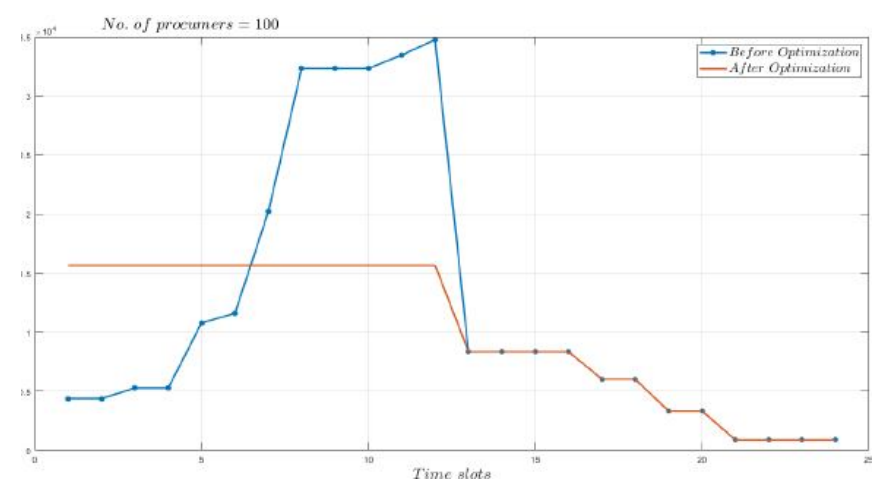

Figure 6.1: Total demand with 100 prosumers on the smart grid over 24 time slots

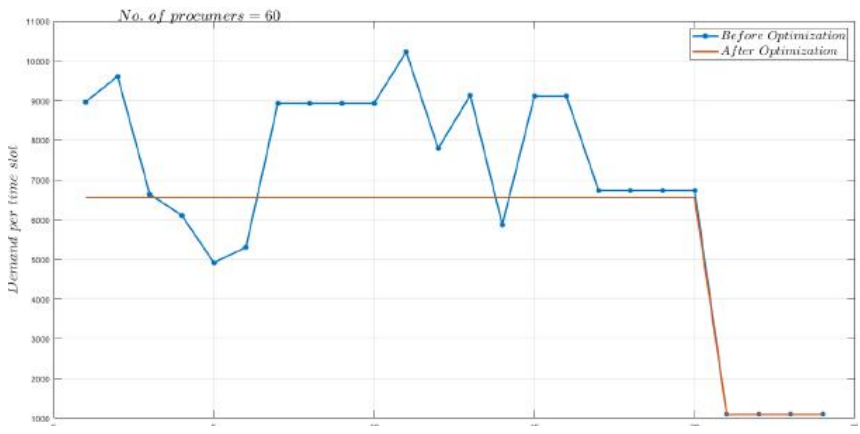

Figure 6.2: Total demand with 60 prosumers on the smart grid over 24 time slots.

As such, transforming into the decentralized system is attend in smart grid to bring more dynamic, intelligent and proactive features. The grid infrastructure itself is also un-der going an adaptation and moving toward a fully automated network having decentralized topologies in order to increase the interactions among all components of smart grid systems in a dynamic way.

Therefore, it is clear from the above simulation results that implementation of blockchain supported demand response can help in regulating load on the smart grid. As stated in chapter II efficiency starts with information flow and the blockchain which maintains records of transactions gives this to both consumers and producers helping them to regulate usage. Another important requirement is participation, this system proposed here can motivate more participation due to cost benets which is shown in Table 5.1 and Table5.2 and additionally participants can obtain incentives for mining which encourages more participation. The microgrid, a grid paradigm, is becoming an integral part of the smart grid which is basically promoted by distributed energy resources (DERs) such as solar, wind and fuel cells. The microgrid is defined as a localized group of DERs, battery storage units, EVs, smart appliances and loads, where the generation units are usually located closed to the loads.

\section{CONCLUSION}

The energy grid is a constantly evolving system in terms of sources with new renewable energy where customers are becoming producers of energy and in terms of technology with the implementation of IoT based smart meters, grid controllers etc. Due to these rapid developments traditional centralized energy systems seems non-viable and unreliable to manage the complex system. Blockchain based smart grids is the current projected future for energy grid. But with all the advantages of this technology comes challenges, some of which were addressed in this work. One of the solutions to the well-known scalability issue of blockchain is lightning network but application of this solution to a network consisting of a physical energy transaction and corresponding monetary transaction has been discussed in this work. A 2 of 3 mortising wallet implemented using lightning network protocol with the utility company being part but not solve custodian of transactions makes it possible to integrate the two different transactions. ever application of the penalty function discussed here discourages defaulters and improves security. The proposed network encourages prosumer participation in terms of incentives from mining, security of commodity by penalty function for prosumers and utility company, who maintains the physical network, and transparency on transaction information. These information paves a better path to implement a DR system which is a key property of smart grids. In this work we have further modeled an optimal demand response system capable of increasing efficiency of smart grid and at the same time bring pros to all network participants. 


\section{REFERENCES}

1. K. Wang, J. Yu, Y. Yu, Y. Qian, D. Zeng, S. Guo, YongXing, and J. Wu, \A survey on energy internet: Architecture, approach, and emerging technologies," IEEE Syst. J., vol. 12, no. 3, pp. 2403-2416, Sep. 2018.

2. X. Fang, S. Misra, G. Xue, and D. Yang, ISmart grid the new and improved power grid: A survey," IEEE Communications Surveys \& tutorials, vol. 14, pp. 944 980, Dec. 2011.

3. Z. Fan, P. Kulkarni, S. Gormus, C. Efthymiou, G. Kalogridis, M. Sooriya bandara, Z. Zhu, S. Lambotharan, and W. Hau Chin, ISmart grid communications: Overview of research challenges, solutions, and standardization activities," IEEE Communications Surveys \& Tutorials, vol. 15, no. 1, pp. 21 - 38, Jan. 2013.

4. D. Alahakoon and X. Yu, Smart electricity meter data intelligence for future energy systems: A survey," IEEE Transactions on Industrial Informatics, vol. 12, no. 1, pp. 425 436, Feb. 2016.

5. R.D.Z.Y.M.-Y.C.J. Chen, A survey on demand response in smart grids: Mathe-matical models and approaches," IEEE Transactions on Industrial Informatics, vol. 11, no.3, pp.570-582, Jun 2015.

6. Y. G. Y. C. Y. G. Yuguang Fang, A privacy-preserving scheme for incentive-based demand response in the smart grid," IEEE Transactions on Smart Grid, vol. 7, no. 3, pp. 1304 - 1313, Mar. 2016.

7. M. Andoni et al., Blockchain technology in the energy sector: A systematic review of challenges and opportunities," Renew. Sust. Energy Rev., vol. 100, pp. 143- 174, Feb.2019.

8. P. Siano, G. De Marco, and A. Rolan and Vincenzo Loia, A survey and evaluation of the potentials of distributed ledger technology for peer-to-peer transactive energy exchanges in local energy markets," IEEE Systems Journal, vol. 13, no. 3, pp. 3454-3466, Sep. 2019.

9. P. Siano, Demand response and smart grids- a survey," Renew. Sustain. Energy Rev., vol. 30, pp. 461-478, Nov. 2013.

10. Z. Zhao, L. Wu, and G. Song, Convergence of volatile power markets with price-based demand response," IEEE Transactions on Power Systems, vol. 29, no. 5, pp. 2107- 2118, Sep. 2014.

11. Peter Palensky and Dietmar Dietrich, Demand side management: Demand response, intelligent energy systems, and smart loads, IEEE Transactions on Industrial Informatics, vol. 7, no. 3, pp. 381-388, Aug. 2011.

12. Shahab Bahrami and Aras Sheikhi, FFrom demand response in smart grid toward integrated demand response in smart energy hub," IEEE Transactions on Industrial Informatics, vol. 7, no. 2, pp. 650\{658, Mar. 2016.

13. YeYang, H. Sharif, and D. Tipper, A survey on smart grid communication infrastructures: Motivations, requirements and challenges," IEEE Communications Surveys \& tutorials, vol. 15, no.1, pp. 5-20, Feb. 2012.

14. R. Ma, H.-H. Chen, Y.-R. Huang, and W. Meng, Smart grid communication: Its challenges and opportunities," IEEE Transactions on Smart Grid, vol. 4, no. 1, pp. 36\{46, Mar. 2013.

15. J. Zhou, R. Q. Hu, and Y. Qian, Scalable distributed communication architectures to support advanced metering infrastructure in smart grid," IEEE Transactions on Parallel and Distributes Systems, vol. 23, no. 9, pp. 1632\{1642, Sep. 2012.

16. T. Moverman, R. W. Sackman, and T. L. Davis, High-assurance smart grid: A three- part model for smart grid control systems," Proceedings of the IEEE, vol. 99, no. 6, pp. 1046-1062, Jun. 2011.

17. V. Namboodiri, V. Aravinthan, S. N. Mohaptra, B. Karimi, and W. Jewell, IToward a secure wireless-based home area network for metering in smart grids," IEEE Systems Journal, vol. 8, no. 2, pp. 509-520, Jun. 2014.

18. Aitzhan, N.Z., Svetinovic, D.: Security and privacy in decentralized energy trading bthrough multi-signatures, blockchain and anonymous messaging streams. IEEE Trans. Dependable Secure Comput. 15(5), 840-852 2018.

19. Andoni, M., et al.: Blockchain technology in the energy sector: a systematic review of challenges and opportunities. Renew. Sustain. Energy Rev. 100, 143-174 (2019).

20. Castellanos, J.A.F., Coll-Mayor, D., Notholt, J.A.: Cryptocurrency as guarantees of origin: Simulating a green certificate market with the ethereum blockchain. In: 2017 IEEE International Conference on Smart Energy Grid Engineering (SEGE), August 2017, pp. 367-372 2017.

21. Fraunhofer Institute for Solar Energy Systems: Photovoltaics report 2018.

22. Guan, Z., et al.: Privacy-preserving and efficient aggregation based on blockchain for power grid communications in smart communities. IEEE Commun. Mag. 56(7), 82-88 2018.

23. Mannaro, K., Pinna, A., Marchesi, M.: Crypto-trading: Blockchain-oriented energy market. In: 2017 AEIT International Annual Conference, September 2017, pp. 1-5 2017.

24. Marchesi, M., Marchesi, L., Tonelli, R.: An agile software engineering method to design blockchain applications. In: Proceedings of the 14th Central and Eastern European Software Engineering Conference Russia, p. 3. ACM 2018

25. Mengelkamp, E., G*arttner, J., Rock, K., Kessler, S., Orsini, L., Weinhardt, C.: Designing microgrid energy markets: a case study: the brooklyn microgrid. Appl. Energy 210, 870-880 2018.

26. Mengelkamp, E., Notheisen, B., Beer, C., Dauer, D., Weinhardt, C.: A blockchainbased smart grid: towards sustainable local energy markets. Comput. Sci. Res. Dev. 33, 207-214 2017. 
27. Neha"1, Z., Guerard, G.: Integration of the blockchain in a smart grid model. In: Proceedings of the 14th International Conference of Young Scientists on Energy Issues, CYSENI 2017, Kaunas, Lithuania, pp. 25-26 2017

28. Pee, S.J., Kang, E.S., Song, J.G., Jang, J.W.: Blockchain based smart energy trading platform using smart contract. In: 2019 International Conference on Artificial Intelligence in Information and Communication (ICAIIC), February 2019, pp. 322- 325.

29. Pop, C., Cioara, T., Antal, M., Anghel, I., Salomie, I., Bertoncini, M.: Blockchain based decentralized management of demand response programs in smart energy grids. Sensors 18(1) 2018.

30. Porru, S., Pinna, A., Marchesi, M., Tonelli, R.: Blockchain-oriented software engineering: challenges and new directions. In: 2017 IEEE/ACM 39th International Conference on Software Engineering Companion (ICSE-C), pp. 169-171. IEEE 2017

31. Richter, B., Mengelkamp, E., Weinhardt, C.: Maturity of blockchain technology in local electricity markets. In: 2018 15th International Conference on the European Energy Market (EEM), June 2018, pp. 1-6 2018.

32. Neha" $1, Z$., Guerard, G.: Integration of the blockchain in a smart grid model. In: Proceedings of the 14th International Conference of Young Scientists on Energy Issues, CYSENI 2017, Kaunas, Lithuania, pp. 25-26 2017.

33. Ashari, Firmansyah. (2020). Smart Contract and Blockchain for Crowdfunding Platform. International Journal of Advanced Trends in Computer Science and Engineering.9.3036-3041.10.30534/ijatcse/2020/83932

020 . 